\title{
Intrinsic Stokes parameters for 3D and 2D polarization states
}

\author{
J. J. Gil \\ ppgil@unizar.es
}

Universidad de Zaragoza, Pedro Cerbuna 12, 50009 Zaragoza, Spain

The second-order characterization of a three-dimensional (3D) state of polarization is provided either by the corresponding $3 \mathrm{D}$ coherency matrix or (equivalently) by the associated 3D Stokes parameters. The analysis of the polarization properties that are invariant under orthogonal transformations of the laboratory reference frame allows to define a set of six intrinsic Stokes parameters which provides a simplified interpretation of $3 \mathrm{D}$ states of polarization in terms of meaningful physical properties. The rotationally invariant properties of $2 \mathrm{D}$ states of polarization are straightforwardly retrieved in a consistent way, so that the $2 \mathrm{D}$ intrinsic Stokes parameters are constituted by the intensity, the degree of linear polarization and the degree of circular polarization.

[DOI: http://dx.doi.org/10.2971/jeos.2015.15054]

Keywords: Physical optics, ellipsometry, polarimetry, polarization, scattering

\section{INTRODUCTION}

The second-order polarization properties of an electromagnetic wave, at a given point $\mathbf{r}$ in the space, are completely determined by a set of nine 3D Stokes parameters [1], which become the customary four 2D Stokes parameters when the components of the electric field of the wave lie in a fixed plane that is taken to represent the two effective components. Unlike conventional 2D Stokes parameters, 3D Stokes parameters have not a straightforward interpretation for arbitrary states (in general with fluctuating direction of propagation). The aim of this work is to define and interpret a set of six "intrinsic Stokes parameters", which summarize those polarization properties that are invariant under arbitrary changes of the laboratory reference frame. It is found that the said intrinsic parameters have direct and meaningful physical interpretation.

The 3D polarization matrix (or coherency matrix) $\mathbf{R}$ is defined as

$$
\mathbf{R}=\left\langle\varepsilon(t) \otimes \varepsilon^{\dagger}(t)\right\rangle
$$

where $\varepsilon(t)$ is the 3D instantaneous Jones vector (whose three components are the analytic signals of the electric field of the wave), $\otimes$ stands for the Kronecker product, the superscript ${ }^{\dagger}$ denotes conjugate transposed, and the brackets indicate time averaging over the measurement time.

Moreover, the space-time coherence-polarization properties of a given 3D electromagnetic wave are appropriately represented by the $3 \mathrm{D}$ coherence-polarization matrix

$$
\mathbf{J}\left(\mathbf{r}_{1}, \mathbf{r}_{2}, \tau\right)=\left\langle\varepsilon\left(\mathbf{r}_{1}, t\right) \otimes \varepsilon^{\dagger}\left(\mathbf{r}_{2}, t+\tau\right)\right\rangle
$$

whose elements are the second order moments of the analytic signals of the field variables taken at points $\mathbf{r}_{1}$ and $\mathbf{r}_{2}$ and at times $t$ and $t+\tau$.
Unitary transformations play different roles with respect to coherence and polarization properties. Any unitary transformation preserves the correlations between the field variables at points $\mathbf{r}_{1}$ and $\mathbf{r}_{2}$, and times $t$ and $t+\tau$. Overall measures of these correlations are given by invariant degrees of coherence like the electromagnetic degree of coherence [2] or the intrinsic degrees of coherence [3].

Unitary transformations can be classified into a) nonorthogonal, which, as shown below, have necessarily to be associated to changes of the polarization state, and b) orthogonal, which always admit their interpretation as changes of the reference frame (rotation in the 3D space), hence preserving those polarization properties that are independent of the laboratory reference frame considered.

To clarify the above distinction, let us consider a light beam with fixed direction of propagation $Z$ (i.e., a $2 \mathrm{D}$ state of polarization), and a reference frame $X Y Z$, and note that while the azimuth of the average polarization ellipse depends on the particular choice of the (mutually orthogonal) directions $X$ and $Y$, the intensity, the degree of polarization and the ellipticity of the average polarization ellipse are independent of arbitrary rotations of the axes $X Y$ about the propagation direction $Z$. These rotations in the real space correspond to rotations of the Poincaré sphere about the $S_{3}$ axis (usually represented vertically) that goes from left-circular to right-circular polarization states. Moreover, nonorthogonal unitary transformations of a 2D coherency matrix (which can physically be realized through interposing retarders with non circular eigenstates in the pathway of the beam) correspond to rotations of the Poincaré sphere that modify the $S_{3}$ axis and cannot physically be realized, in any way, as rotations of the laboratory reference frame. In other words, nonorthogonal unitary transformations of a $2 \mathrm{D}$ state of polarization necessarily af- 
fect the ellipticity, which is an intrinsic attribute of a given 2D state. Analogously, nonorthogonal unitary transformations of the coherency matrix of a 3D state can never be physically realized through rotations of the laboratory reference frame (i.e., $3 \mathrm{D}$ rotations in the real space).

Rotations of the laboratory reference frame $X Y Z$ are represented by $3 \times 3$ proper orthogonal matrices $Q$, so that the transformed coherency matrix $\mathbf{R}^{\prime}$ representing the same state as $\mathbf{R}$ but referred to the new reference frame, is given by $\mathbf{R}^{\prime}=\mathbf{Q R}^{T}$. As proposed by Dennis [4] it is worth to consider the decomposition of $\mathbf{R}$ into its real and imaginary parts $\mathbf{R}=\mathbf{R}_{R}+i \mathbf{R}_{I}$, where the real matrix $\mathbf{R}_{R} \equiv \operatorname{Re}(\mathbf{R})$ is symmetric and positive-semidefinite, while the imaginary component $\mathbf{R}_{I} \equiv \operatorname{Im}(\mathbf{R})$ is skew-symmetric $\left(\mathbf{R}_{I}=-\mathbf{R}_{I}^{T}\right)$. $\mathbf{R}_{R}$ can always be diagonalized through a particular rotation $\mathbf{Q}_{O}$ of the reference frame [4]

$$
\mathbf{Q}_{O} \mathbf{R}_{R} \mathbf{Q}_{O}^{T}=\operatorname{diag}\left(a_{1}, a_{2}, a_{3}\right) \quad\left(0 \leq a_{3} \leq a_{2} \leq a_{1}\right)
$$

Since $\mathbf{R}_{R}$ is a positive-semidefinite symmetric matrix, it defines an ellipsoid [5], centered at the origin, whose semiaxes $\left(a_{1}, a_{2}, a_{3}\right)$ lie along the respective transformed axes $X_{O} Y_{O} Z_{O}$. As pointed out in reference [6], the diagonal matrix $\operatorname{diag}\left(a_{1}, a_{2}, a_{3}\right)$ can be interpreted as the coherency matrix of a state composed of the incoherent superposition of three linearly polarized pure states

$$
\begin{aligned}
\operatorname{diag}\left(a_{1}, a_{2}, a_{3}\right) & =\mathbf{R}_{p 1}+\mathbf{R}_{p 2}+\mathbf{R}_{p 3} \\
\mathbf{R}_{p 1} & \equiv a_{1} \operatorname{diag}(1,0,0) \\
\mathbf{R}_{p 2} & \equiv a_{2} \operatorname{diag}(0,1,0) \\
\mathbf{R}_{p 3} & \equiv a_{3} \operatorname{diag}(0,0,1)
\end{aligned}
$$

with respective intensities $a_{1}, a_{2}$ and $a_{3}$.

By applying the rotation $\mathbf{Q}_{O}$ to the whole matrix $\mathbf{R}$ we get the transformed coherency matrix [4]

$$
\begin{aligned}
& \mathbf{R}_{O}=\operatorname{diag}\left(a_{1}, a_{2}, a_{3}\right)+i \mathbf{R}_{n}, \\
& \mathbf{R}_{n} \equiv\left(\begin{array}{ccc}
0 & -n_{O 3} & n_{O 2} \\
n_{O 3} & 0 & -n_{O 1} \\
-n_{O 2} & n_{O 1} & 0
\end{array}\right)
\end{aligned}
$$

The orthogonal matrix $\mathbf{Q}_{O}$ can always be expressed as the following product of rotations around the respective axes ZYX

$$
\mathbf{Q}_{O}=\mathbf{Q}_{Z}(\phi) \mathbf{Q}_{Y}(\beta) \mathbf{Q}_{X}(\alpha)
$$

The rotation $\mathbf{Q}_{Z}(\phi)$ around the $Z$ axis has been chosen in the indicated position in order to make easier the recovery of $2 \mathrm{D}$ states (i.e. states with fixed direction of propagation) embedded into the 3D representation. Obviously, any alternative sequence of the rotations is also physically admissible and realizable. Thus, $\mathbf{R}$ can be parameterized through the following nine independent parameters: the three orientation angles $(\phi, \alpha, \beta)$; the semiaxes of the intensity ellipsoid, or inertia ellipsoid [4], given by the principal intensities $\left(a_{1}, a_{2}, a_{3}\right)$, and the three components $\left(n_{\mathrm{O} 1}, n_{\mathrm{O} 2}, n_{\mathrm{O} 3}\right)$ of the angular momentum $\mathbf{n}_{\mathrm{O}}$ of the wave along the respective axes $X_{O} Y_{O} Z_{O}$. The intensity $I$ of the state $\mathbf{R}_{O}$ is given by

$$
I=\operatorname{tr} \mathbf{R}=\operatorname{tr} \mathbf{R}_{O}=a_{1}+a_{2}+a_{3}
$$

The intrinsic coherency matrix $\mathbf{R}_{O}$ [6] represents the same state as that represented by $\mathbf{R}$ but referred to the intrinsic coordinate frame $X_{O} Y_{O} Z_{O}$. The smaller is the third principal intensity $a_{3}$, the smaller is the solid angle around the axis $Z_{O}$ that limits the range of compatible orientations of $\mathbf{n}_{O}$. When $a_{3}=0, \mathbf{n}_{O}$ is forced to lie along the axis $Z_{O}$, which, in turn, in this case is precisely the well-defined direction of propagation of the state $\mathbf{R}_{O}$.

Up to the limits set by the restrictive inequalities derived from the non-negativity of $\mathbf{R}_{O}$ [4], the quantities $\left(a_{1}, a_{2}, a_{3} ; n_{\mathrm{O} 1}, n_{\mathrm{O} 2}, n_{\mathrm{O} 3}\right)$ are independent and are intrinsic of a given coherency matrix $\mathbf{R}$. We stress the fact that the only unitary transformations of $\mathbf{R}$ that preserve the state of polarization (in the sense described in the introductory paragraphs) are those that correspond to rotations of the Cartesian reference frame (i.e., orthogonal transformations), and consequently $\mathbf{R}_{O}$ (and hence $\mathbf{R}$ ) involves six polarization invariant parameters, beyond the only three physical invariants derivable from the eigenvalues of $\mathbf{R}$.

The 3D Stokes parameters are defined as $s_{k}=\operatorname{tr}\left(\mathbf{R} \boldsymbol{\omega}_{k}\right)$, where $\omega_{k}$ are the Gell-Mann matrices (including the identity matrix $\left.\omega_{0}\right)$ or (equivalently) as

$$
s_{i j}=\operatorname{tr}\left(\mathbf{R} \omega_{i j}\right)
$$

where, in order to emphasize symmetry properties, the GellMann matrices have been labeled as follows

$$
\begin{gathered}
\omega_{00} \equiv\left(\begin{array}{lll}
1 & 0 & 0 \\
0 & 1 & 0 \\
0 & 0 & 1
\end{array}\right), \quad \omega_{01} \equiv\left(\begin{array}{ccc}
0 & 1 & 0 \\
1 & 0 & 0 \\
0 & 0 & 0
\end{array}\right), \\
\omega_{02} \equiv\left(\begin{array}{lll}
0 & 0 & 1 \\
0 & 0 & 0 \\
1 & 0 & 0
\end{array}\right), \quad \omega_{10} \equiv\left(\begin{array}{ccc}
0 & -i & 0 \\
i & 0 & 0 \\
0 & 0 & 0
\end{array}\right), \\
\omega_{11} \equiv\left(\begin{array}{ccc}
1 & 0 & 0 \\
0 & -1 & 0 \\
0 & 0 & 0
\end{array}\right), \quad \omega_{12} \equiv\left(\begin{array}{ccc}
0 & 0 & 0 \\
0 & 0 & 1 \\
0 & 1 & 0
\end{array}\right), \\
\omega_{20} \equiv\left(\begin{array}{ccc}
0 & 0 & -i \\
0 & 0 & 0 \\
i & 0 & 0
\end{array}\right), \quad \omega_{21} \equiv\left(\begin{array}{ccc}
0 & 0 & 0 \\
0 & 0 & -i \\
0 & i & 0
\end{array}\right), \\
\omega_{22} \equiv \frac{1}{\sqrt{3}}\left(\begin{array}{ccc}
1 & 0 & 0 \\
0 & 1 & 0 \\
0 & 0 & -2
\end{array}\right),
\end{gathered}
$$

so that $s_{i j}$ can be considered as the respective elements of the Stokes-parameter matrix.

\section{INTRINSIC STOKES PARAMETERS}

The intrinsic coordinate frame $X_{O} Y_{O} Z_{O}$ provides a simplified and meaningful view of the physically invariant quantities that characterize a polarization state. In particular, the six intrinsic Stokes parameters are defined as the 3D Stokes parameters $t_{k}=\operatorname{tr}\left(\mathbf{R}_{O} \boldsymbol{\omega}_{k}\right)$ associated with $\mathbf{R}_{O}$, or, by using the double subscript notation, as

$$
t_{i j}=\operatorname{tr}\left(\mathbf{R}_{O} \boldsymbol{\omega}_{i j}\right)
$$


so that $t_{i j}$ are the elements of the left-triangular intrinsic Stokesparameter matrix

$$
\mathbf{T}=\left(\begin{array}{ccc}
a_{1}+a_{2}+a_{3} & 0 & 0 \\
2 n_{O 3} & a_{1}-a_{2} & 0 \\
-2 n_{O 2} & 2 n_{O 1} & \left(a_{1}+a_{2}-2 a_{3}\right) / \sqrt{3}
\end{array}\right)
$$

which can also be expressed as

$$
\mathbf{T}=I\left(\begin{array}{ccc}
1 & 0 & 0 \\
2 \hat{n}_{O 3} & P_{l} & 0 \\
-2 \hat{n}_{O 2} & 2 \hat{n}_{O 1} & P_{d} / \sqrt{3}
\end{array}\right)
$$

where $\hat{n}_{O l} \equiv n_{O l} / I(l=1,2,3)$ are the components of the normalized angular momentum vector $\hat{\mathbf{n}}_{O} \equiv \mathbf{n}_{O} / I$. The notation used for the diagonal elements in Eq. (12) is justified in the following paragraphs.

In light of the above expressions, we achieve an interpretation of the intrinsic Stokes parameters by means of the following considerations:

- As expected, $t_{00} \equiv a_{1}+a_{2}+a_{3}$ is the intensity $I$. Obviously, the only restriction on the value of $t_{00}$ is that it is nonnegative.

- $t_{11} \equiv a_{1}-a_{2}$ is the difference between the major and the intermediate semiaxes of the intensity ellipsoid, i.e., the difference between the intensities of the linear components along respective axes $X_{O}$ and $Y_{O}$. The normalized parameter $\hat{t}_{11} \equiv t_{11} / I \equiv P_{l}$ gives a measure of the relative portion of the power of the electromagnetic wave that can be allocated as linearly polarized and, as shown in reference [6], can properly be called the degree of linear polarization. The maximum $P_{l}=1\left(t_{11}=t_{00}\right)$ is achieved for linearly polarized pure states. The minimum $P_{l}=0\left(t_{11}=0\right)$ corresponds to states constituted by arbitrary incoherent combinations of 3D unpolarized states (i.e. fully random 3D states) and circularly polarized pure states.

- $t_{22}$ is $1 / \sqrt{3}$ times the difference between the sum of intensities of the linear components lying on the plane $X_{O} Y_{O}$ and twice the intensity of the component lying on the axis $Z_{O}$. The normalized parameter $\hat{t}_{22} \equiv t_{22} / I \equiv P_{d} / \sqrt{3}$ gives a measure of the relative portion of the power of the electromagnetic wave that corresponds to the combined contributions of the components lying on the plane $X_{O} Y_{O}$ minus twice the contribution of the component lying on the axis $Z_{O}$. The quantity $\hat{t}_{22}$ is particularly important for the interpretation of the 3D state of polarization because it provides an appropriate measure for its degree of directionality $P_{d} \equiv \sqrt{3} \hat{s}_{O 22}$ [6] (i.e. the lack of randomness of the direction of propagation). The equality $P_{d}=1$ (i.e., $\sqrt{3} s_{\mathrm{O} 22}=s_{\mathrm{O} 00}$ ) is satisfied by states whose fluctuating polarization ellipse remains in the fixed plane determined by $X_{O} Y_{O}\left(a_{3}=0\right)$. Moreover, $P_{d}=0$ is satisfied by $3 \mathrm{D}$-unpolarized states.

- The intrinsic representation of the spin angular momentum of the wave is given by the vector $\mathbf{n}_{O} \equiv\left(t_{21},-t_{20}, t_{10}\right)^{T}$, determined by the three offdiagonal intrinsic Stokes parameters. Since we are considering the polarization properties at a fixed point $\mathbf{r}$ in the space and not an extensive portion of the wavefront, the orbital angular momentum does not contribute to $\mathbf{n}_{O}$.

- In analogy to the case of two-dimensional states of polarization, we can define the 3D degree of circular polarization as the following quadratic average of the off-diagonal intrinsic Stokes parameters

$$
P_{c} \equiv \sqrt{\hat{t}_{21}^{2}+\hat{t}_{20}^{2}+\hat{t}_{10}^{2}}=2 \hat{n}
$$

The maximum value of $\hat{n}, \hat{n}_{\max }=1 / 2$, is achieved for pure circularly polarized states, while the minimum $\hat{n}_{\min }=0$ corresponds to states constituted by incoherent mixtures of three components linearly polarized along the respective axes $X_{O} Y_{O} Z_{O}$ (with respective intensities $\left.a_{1}, a_{2}, a_{3}\right)$.

- The 3D degree of polarimetric purity $P_{3 \mathrm{D}}[7,8]$ (also called 3D degree of polarization [9]-[15]), defined as

$$
P_{3 \mathrm{D}}=\sqrt{\frac{1}{2}\left(\frac{3 \operatorname{tr}\left(\mathbf{R}^{2}\right)}{(\operatorname{tr} \mathbf{R})^{2}}-1\right)}
$$

is given by the following quadratic average of the six normalized intrinsic Stokes parameters

$$
\begin{aligned}
P_{3 \mathrm{D}} & =\frac{\sqrt{3}}{2} \sqrt{\hat{t}_{10}^{2}+\hat{t}_{11}^{2}+\hat{t}_{20}^{2}+\hat{t}_{21}^{2}+\hat{t}_{22}^{2}} \\
& =\frac{\sqrt{3}}{2} \sqrt{P_{l}^{2}+P_{c}^{2}+P_{d}^{2} / 3}
\end{aligned}
$$

so that the sources of polarimetric purity are identified as $P_{d}, P_{l}$ and $P_{c}$, with independent and complementary contributions. Note that, in accordance with Ellis et al. [16,17] we consider preferable to reserve the term degree of polarization for the quantity defined as $P_{1} \equiv\left(\lambda_{1}-\lambda_{2}\right) /(\operatorname{tr} \mathbf{R})$, which is precisely the so-called first index of polarimetric purity $[7,8,18]$. This comment does not restrict the known fact that a complete characterization of the structure of randomness of a 3D state of polarization requires two parameters, as for instance the two indices of polarimetric purity [7].

\section{INTRINSIC STOKES PARAMETERS FOR 2D STATES EMBEDDED INTO THE 3D REPRESENTATION}

In the case of $2 \mathrm{D}$ states, defined by the condition $P_{d}=1$, i.e., states where the electric field vector remains inside a fixed plane, this plane is precisely which contains the axes $X_{O} Y_{O}$ of the intrinsic coordinate frame, and therefore the angular momentum vector lies along the axis $Z_{O}$ $\left(t_{20}=t_{21}=0, \sqrt{3} t_{22}=I\right)$. The $2 \mathrm{D}$ representation is retrieved through the $2 \mathrm{D}$ coherency matrix embedded into the upper-left $2 \times 2$ submatrix of $\mathbf{R}_{O}$

$$
\begin{aligned}
\mathbf{R}_{O} & =\frac{1}{2}\left(\begin{array}{ccc}
t_{00}+t_{11} & -i t_{10} & 0 \\
i t_{10} & t_{00}-t_{11} & 0 \\
0 & 0 & 0
\end{array}\right) \\
& =\frac{I}{2}\left(\begin{array}{ccc}
1+P_{l} & -i P_{c} & 0 \\
i P_{c} & 1-P_{l} & 0 \\
0 & 0 & 0
\end{array}\right)
\end{aligned}
$$


and the corresponding 2D intrinsic Stokes vector $\mathbf{t}$ is given by

$$
\mathbf{t}=I\left(1, P_{l}, 0, P_{c}\right)^{T}
$$

The degree of polarization for 2D states is retrieved as

$$
P=\sqrt{\hat{t}_{11}^{2}+\hat{t}_{10}^{2}}=\sqrt{P_{l}^{2}+P_{c}^{2}}
$$

The general 2D Stokes parameters referred to an arbitrary reference frame $X Y Z_{O}$ are obtained through an arbitrary rotation around the axis $Z_{O}$ (orthogonal to the plane containing the fluctuating electric field of the wave).

The general 3D representation of a 2D state is obtained by applying an arbitrary rotation $\mathbf{R}=\mathbf{Q}^{T} \mathbf{R}_{O} \mathbf{Q}$ transforming the intrinsic axes $X_{O} Y_{O} Z_{O}$ to other coordinate axes $X Y Z$, so that the angular momentum vector is given by

$$
\mathbf{n} \equiv \frac{1}{2}\left(s_{21},-s_{20}, s_{10}\right)^{T}
$$

in terms of the Stokes parameters $s_{21}, s_{20}$ and $s_{10}$. Obviously, the absolute value of $\mathbf{n}$ is invariant with respect to orthogonal transformations:

$$
\begin{aligned}
n \equiv|\mathbf{n}| & =\frac{1}{2} \sqrt{s_{21}^{2}+s_{20}^{2}+s_{10}^{2}} \\
& =\frac{1}{2} \sqrt{t_{21}^{2}+t_{20}^{2}+t_{10}^{2}}=\frac{I}{2} P_{c}
\end{aligned}
$$

The direction $\hat{\mathbf{n}}$ (along which the electric field has zero projection, Figure 1) is given by $x / s_{21}=-y / s_{20}=z / s_{10}$ [19], while the equation of the plane $\Pi$ within which the electric field evolves is given by $s_{21} x-s_{20} y+s_{10} z=0$ [19].

Note that there are situations where the angular momentum of the $2 \mathrm{D}$ state is zero (i.e., the coherency matrix is real) and the above procedure for determining the plane $\Pi$ cannot be applied. This occurs for states constituted by incoherent combinations of 2D unpolarized states and 2D states whose average polarization ellipse is a straight segment (zero ellipticity). The 3D intrinsic Stokes-parameter matrix of such particular $2 \mathrm{D}$ states has the form $\mathbf{T}=I \operatorname{diag}\left(1, P_{l}, 0\right)$ (with $P_{l}=0$ for unpolarized states) and the angles $\alpha, \beta$ determining the direction normal to the plane $\Pi$ can be obtained from the orthogonal matrix that diagonalizes $\mathbf{R}_{R}$.

In the particular case of a pure linearly polarized state, $\mathbf{T}$ has the form $\mathbf{T}=I \operatorname{diag}(1,1,0)$, and the plane $\Pi$ is defined up to a rotation about the polarization axis $X_{O}$. As shown by Sheppard [19], in this case the direction cosines $\left(c_{1}, c_{2}, c_{3}\right)$ for the polarization axis $X_{O}$ with respect to the arbitrary reference frame $X Y Z$ are expressed as follows in terms of the corresponding 3D Stokes parameters

$$
c_{1}^{2}=\frac{s_{01} s_{20}}{2 s_{00} s_{12}}, \quad c_{2}^{2}=\frac{s_{01} s_{12}}{2 s_{00} s_{20}}, \quad c_{3}^{2}=\frac{s_{20} s_{12}}{2 s_{00} s_{01}} .
$$

Note that the relation $c_{1}^{2}+c_{2}^{2}+c_{3}^{2}=1$ is satisfied because the 3D Stokes parameters of a pure state are related through the restrictive relations derived from the condition $\operatorname{rank} \mathbf{R}_{O}=1$.

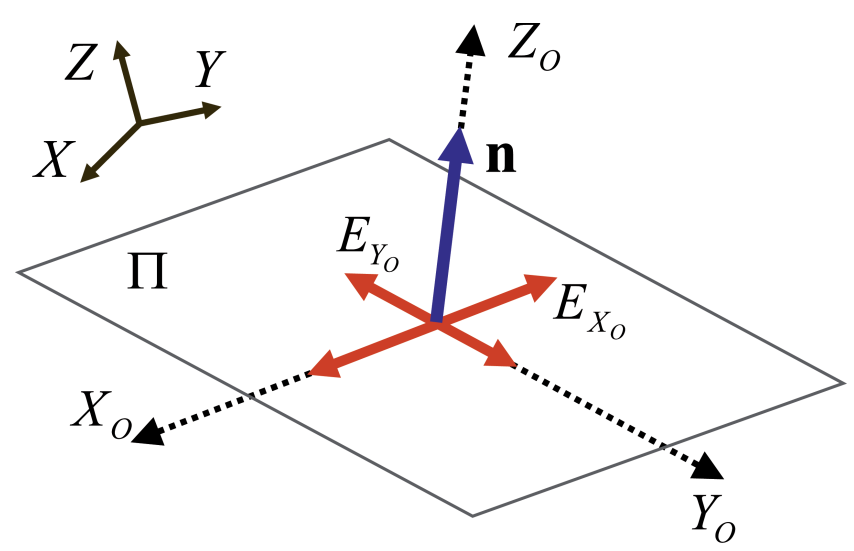

FIG. 1 For a 2D polarization state, the angular momentum $\mathbf{n}$ determines the direction orthogonal to the plane $\Pi$ containing the fluctuating electric field.

\section{ACKNOWLEDGEMENTS}

The author thanks Alfredo Luis for insightful discussions on this subject. This research was supported by Ministerio de Economía y Competitividad and the European Union, grants FIS2011-22496 and FIS2014-58303-P, and by Gobierno de Aragón, group E99.

\section{References}

[1] C. Brosseau, Fundamentals of polarized light: a statistical optics approach (Wiley, New York, 1998).

[2] J. Tervo, T. Setälä, and A. T. Friberg, "Degree of coherence for electromagnetic fields," Opt. Express 11, 1137-1143 (2003).

[3] P. Réfrégier, and F. Goudail, "Invariant degrees of coherence of partially polarized light," Opt. Express 13, 6051-6060 (2005).

[4] M. R. Dennis, "Ceometric interpretation of the three-dimensional coherence matrix for nonparaxial polarization," J. Opt. A: Pure Appl. Opt. 6, S26-S31 (2004).

[5] S. Banerjee, and A. Roy, Linear algebra and matrix analysis for statistics (CRC Press, Boca Raton, 2014).

[6] J. J. Gil, "Interpretation of the coherency matrix for threedimensional polarization states," Phys. Rev. A 90, 043858 (2014).

[7] J. J. Gil, "Polarimetric characterization of light and media," Eur. Phys. J. Appl. Phys. 40, 1-47 (2007).

[8] I. San José, and J. J. Gil, "Invariant indices of polarimetric purity. Ceneralized indices of purity for $\mathrm{n} \times \mathrm{n}$ covariance matrices," $0 \mathrm{pt}$. Commun. 284, 38-47 (2011).

[9] R. Barakat, "Degree of polarization and the principal idempotents of the coherency matrix," Opt. Commun. 23, 147-150 (1977).

[10] T. Setälä, A. Shevchenko, M. Kaivola, and A. T. Friberg, "Degree of polarization for optical near fields," Phys. Rev. E 66, 016615 (2002).

[11] J. J. Gil, J. M. Correas, P. A. Melero, and C. Ferreira, "Generalized polarization algebra," Monog. Sem. Mat. G. Galdeano 31, 161-167 (2004).

[12] A. Luis, "Degree of polarization for three-dimensional fields as a distance between correlation matrices," Opt. Commun. 253, 10-14 (2005).

[13] A. Luis, "Polarization distribution and degree of polarization for three-dimensional quantum light fields," Phys. Rev. A 71, 063815 (2005). 
[14] T. Setälä, K. Lindfors, and A. T. Friberg, "Degree of polarization in 3D optical fields generated from a partially polarized plane wave," Opt. Lett. 34, 3394-3396 (2009).

[15] J. M. Auñón, and M. Nieto-Vesperinas, "On two definitions of the three-dimensional degree of polarization in the near field of statistically homogeneous partially coherent sources," Opt. Lett. 38, 58-60 (2013).

[16] J. Ellis, A. Dogariu, S. Ponomarenko, and E. Wolf, "Degree of polarization of statistically stationary electromagnetic fields," 0 pt. Commun. 248, 333-337 (2005).
[17] J. Ellis, and A. Dogariu, "On the degree of polarization of random electromagnetic fields," Opt. Commun. 253, 257-265 (2005).

[18] J. J. Gil, and I. San José, "3D polarimetric purity," Opt. Commun. 283, 4430-4434 (2010).

[19] C. J. R. Sheppard, "Jones and Stokes parameters for polarization in three dimensions," Phys. Rev. A 90, 023809 (2014). 